\title{
Isquemia miocárdica: conceptos básicos, diagnóstico e implicaciones clínicas. Tercera parte
}

\author{
Pedro R. Moreno ${ }^{\mathrm{a}}$ y Juan H. del Portillo ${ }^{\mathrm{b}, *}$ \\ a Icahn School of Medicine at Mount Sinai, New York, USA \\ b Cardiología, Fundación Santa Fe de Bogotá, Bogotá, Colombia
}

Recibido el 10 de noviembre de 2015; aceptado el 25 de febrero de 2016

\author{
PALABRAS CLAVE \\ Enfermedad \\ coronaria; \\ Isquemia; \\ Cardiopatía \\ isquémica; \\ Hibernación; \\ Flujo sanguíneo
}

\begin{abstract}
Resumen El término «cardiopatía isquémica» se refiere a la disfunción del ventrículo izquierdo secundaria a infarto del miocardio, miocardio isquémico viable o enfermedad coronaria severa documentada por arteriografía coronaria, la cual tiene un pobre pronóstico, con una supervivencia del $45 \%$ a 5 años. El tratamiento de la cardiopatía isquémica involucra la estimación de la viabilidad en el miocardio afectado para determinar si la revascularización puede generar una remodelación positiva que mejore la función del ventrículo izquierdo. Existen cuatro modalidades básicas usadas en la práctica clínica para calcular la viabilidad miocárdica: tomografía de emisión simple de positrones, tomografía por emisión de positrones, ecocardiograma estrés y resonancia magnética cardiaca. Hoy en día hay estudios que demuestran que la terapia médica mejora la función del ventrículo izquierdo en la cardiopatía isquémica, independiente de la presencia o no de viabilidad o de la revascularización miocárdica; por tanto es posible que otros factores como la cantidad de remodelado, los volúmenes del ventrículo izquierdo, la insuficiencia mitral y la fracción de eyección puedan afectar también los desenlaces. Se requiere definir de manera clara los estadios del remodelado ventricular izquierdo en los cuales la presencia de viabilidad es benéfica y las etapas en las que el remodelado es reversible con la revascularización miocárdica. En cuanto a los métodos para determinar la viabilidad, la resonancia magnética parece dar más respuestas al respecto, ya que puede aportar información adicional relacionada con dimensiones del ventrículo izquierdo, fracción de eyección, fibrosis miocárdica y anormalidades valvulares.

(C) 2016 Sociedad Colombiana de Cardiología y Cirugía Cardiovascular. Publicado por Elsevier España, S.L.U. Este es un artículo Open Access bajo la licencia CC BY-NC-ND (http:// creativecommons.org/licenses/by-nc-nd/4.0/).
\end{abstract}

\footnotetext{
* Autor para correspondencia.

Correo electrónico: drdelportillo@gmail.com (J.H. del Portillo).
} 


\section{KEYWORDS}

Coronary disease;

Ischemia;

Ischemic cardiac

pathology;

Hibernation;

Blood flow

\section{Myocardial ischemia: basic concepts, diagnosis and clinical implications. Part three}

\begin{abstract}
The term myocardial ischemia refers to a left ventricular dysfunction secondary to a myocardial infarction, viable ischemic myocardium or sever coronary disease documented by means of a coronary angiography, which has a poor prognosis, with five-year survival rate of $45 \%$. Management of myocardial ischemia involves estimating viability of the affected myocardium in order to determine whether revascularization can generate a positive remodelling that improves left ventricle functioning. Four different basic modalities are used in clinical practice to assess myocardial viability: single-photon emission computed tomography, positron emission tomography, stress echocardiogram and cardiac magnetic resonance. Nowadays there are studies that have shown medical therapy improves left ventricle function in ischemic heart disease, regardless of the presence or not of the viability or the myocardial revascularization; therefore, it is possible that other factors such as the amount of remodelling, the left ventricle volumes, mitral insufficiency and ejection fraction could also afffect the outcomes. A clear definition of the left ventricle remodelling states where the presence of viability is beneficial and the stages where the remodelling is reversible with myocardial revascularization is required. With regards to methods for assessing viability, magnetic resonance seems to provide more answers, as it can give additional information related to the dimensions of the left ventricle, ejection fraction, myocardial fibrosis and valvular abnormalities.

(c) 2016 Sociedad Colombiana de Cardiología y Cirugía Cardiovascular. Published by Elsevier España, S.L.U. This is an open access article under the CC BY-NC-ND license (http:// creativecommons.org/licenses/by-nc-nd/4.0/).
\end{abstract}

\section{Cardiopatía isquémica}

El término se refiere a la disfunción del ventrículo izquierdo secundario a infarto del miocardio, miocardio isquémico viable o enfermedad coronaria severa documentada por arteriografía coronaria. Una definición más estricta es la de Felker et al., que aplica para propósitos de investigación clínica, y se explica como pacientes con disfunción del ventrículo izquierdo e historia de infarto del miocardio o revascularización miocárdica en el pasado, estenosis mayor al $75 \%$ de la coronaria izquierda principal o descendente anterior proximal, o más del $75 \%$ de estenosis de dos o más vasos epicárdicos ${ }^{1}$. Por esto, el término de cardiopatía isquémica puede ser controvertido pese a ser la causa más común de disfunción del ventrículo izquierdo en países desarrollados ${ }^{2}$. Tiene un pobre pronóstico, con una supervivencia del $45 \%$ a 5 años, en comparación con la cardiopatía no isquémica cuya sobrevida a los 5 años es del $62 \%{ }^{1}$.

El tratamiento involucra el diagnóstico de la enfermedad arterial coronaria y requiere la estimación de la viabilidad en el miocardio afectado, ya que en caso de que esté indicada la revascularización, esta podrá generar una remodelación positiva y mejorar la función del ventrículo izquierdo. Aproximadamente el $60 \%$ de los pacientes con ventrículo izquierdo isquémico disfuncional tienen miocardio viable, de ahí que en la próxima sección se discutirá sobre el cálculo de la viabilidad, la cual se define como la capacidad del miocardio disfuncional para retornar a una función normal luego de la revascularización. Datos recientes demostraron que el cálculo de la viabilidad en pacientes con disfunción del ventrículo izquierdo, afecta el desenlace, sin embargo, la mayoría de estos datos corresponden a estudios retrospectivos y metanálisis ${ }^{3}$ (tabla 1). Los pacientes con viabilidad miocárdica a quienes se les realiza revascularización, tienen menor mortalidad y mejores desenlaces cuando se comparan con aquellos que no tienen evidencia de viabilidad; al parecer, estos últimos no tienen la necesidad de ser revascularizados ya que con solo la terapia médica óptima pueden obtener resultados favorables. Pese a que la revascularización miocárdica en pacientes con viabilidad se asocia con recuperación contráctil, prevención de futuras bajas de fracción de eyección, infartos de miocardio adicionales, progreso de la dilatación del ventrículo izquierdo y muerte súbita cardiaca, la mejoría también depende de la cantidad de miocardio viable, ya que se requiere de al menos un $20 \%$ de viabilidad para obtener un beneficio significativo luego de la revascularización ${ }^{4}$.

\section{Cálculo de la viabilidad miocárdica}

Existen cuatro modalidades básicas usadas en la práctica clínica para calcular la viabilidad miocárdica: tomografía de emisión simple de positrones (SPECT), tomografía por emisión de positrones (PET), ecocardiograma estrés y resonancia magnética cardíaca (RMC). Estas modalidades capturan diferentes propiedades del miocito, como la integridad de la membrana, el metabolismo, la integridad mitocondrial, la evidencia de cicatriz miocárdica y la reserva contráctil.

\section{Tomografía de emisión simple de positrones}

La SPECT es una modalidad ampliamente disponible, bien establecida desde el punto vista clínico y con validación pronóstica. Los trazadores más usados incluyen talio-201 análogo del potasio (201 TL) y tecnecio99 m (99mTC). Estos trazadores serán captados en áreas con perfusión intacta, ya que la captación intramiocárdica luego de la infusión 
Tabla 1 Cálculo de la viabilidad en pacientes con disfunción del ventrículo izquierdo

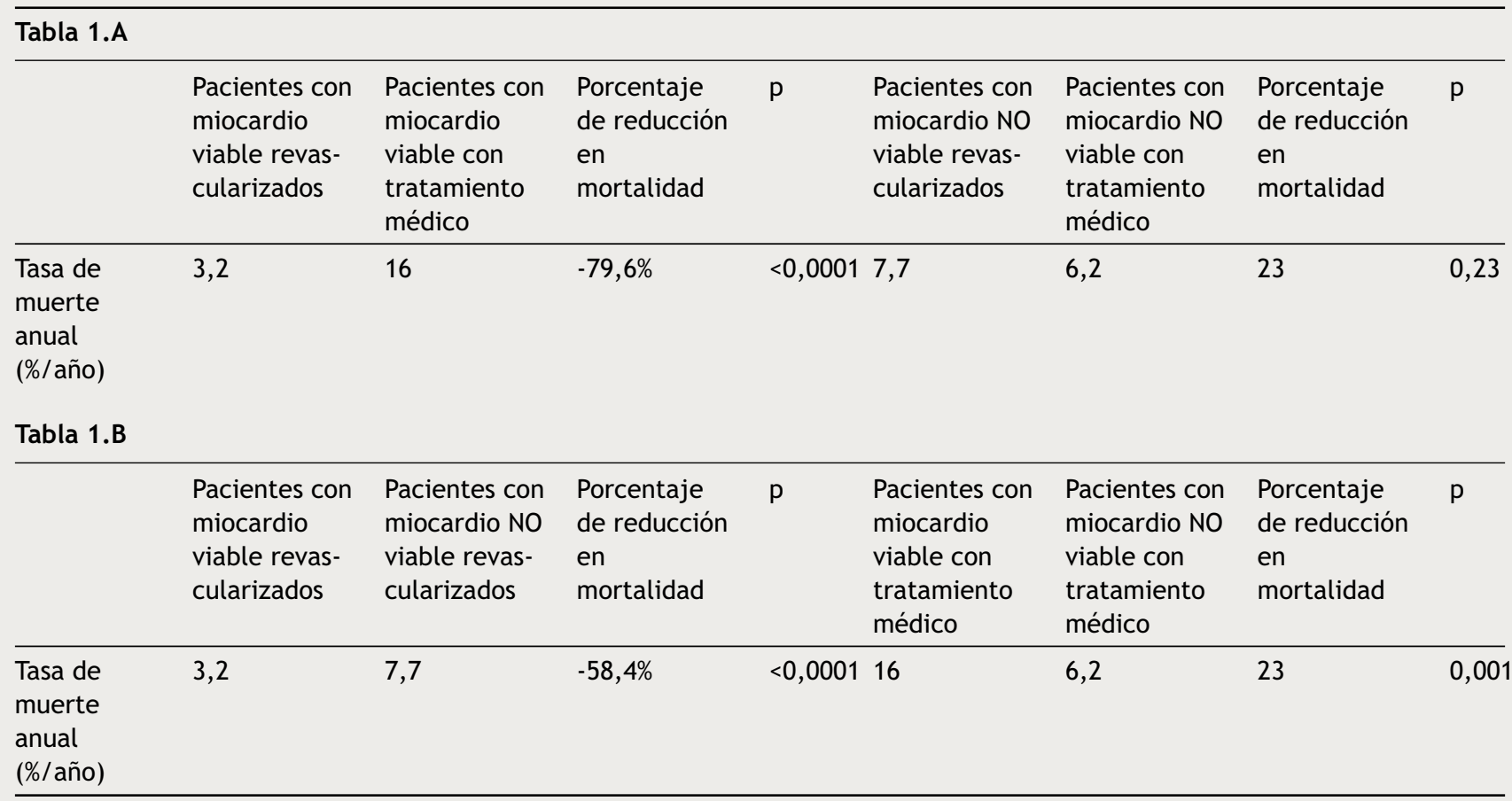

Tabla que representa en (A) Tasa de muertes para pacientes con y sin viabilidad miocárdica tratados con revascularización o con tratamiento médico. Hay un 79,6\% de reducción en mortalidad para pacientes con viabilidad tratados con revascularización ( $\mathrm{p}<0,0001)$. En pacientes sin viabilidad miocárdica, no hubo diferencia significativa de mortalidad con la revascularización versus el tratamiento médico. (B) Los mismos datos que (A) con comparación basada en la estrategia de tratamiento con y sin viabilidad. La mortalidad anual fue baja en pacientes revascularizados con viabilidad frente a los que no tenían viabilidad $(3,2 \%$ vs. $7,7 \%, p<0,0001)$. La mortalidad anual fue significativamente mayor en el grupo de tratamiento médico cuando la viabilidad estaba presente vs. ausente $(16 \%$ vs. $6,2 \%$ $\mathrm{p}=0,001)$. Modificado de: J Am Coll Cardiol. 2002;39(7):1151-83

intravenosa es proporcional al flujo sanguíneo regional, por lo que una disminución en la captación, refleja un compromiso en el suministro de sangre; en este caso el miocardio subyacente podría estar muerto o hibernante. Luego de 21 a 24 horas existe una redistribución de los trazadores basada en el flujo sanguíneo regional, reteniéndose en células con sarcómeras (talio) y mitocondrias (tecnecio) intactas, lo cual refleja viabilidad. Por tanto, si aparece un defecto de captación al inicio de la inyección, pero durante la adquisición tardía esta captación mejora, indica viabilidad miocárdica en presencia de defectos de perfusión. Comúnmente se usan protocolos con talio de reposo-redistribución para evaluar viabilidad, y protocolos de $4 \mathrm{~h}$ de estrés y reinyección para evaluar tanto isquemia como viabilidad. La ventaja del Tecnecio es que tiene menor distribución que el talio, emitiendo factores de alta energía que incrementan la calidad de la imagen, disminuye los artefactos de los tejidos blandos y minimiza la radiación total por la vida media más corta.

Por su parte, el gated-SPECT (imágenes de perfusión sincronizadas con electrocardiograma) tiene ventajas al proveer información de la función del ventrículo izquierdo y viabilidad de forma simultánea; no obstante, las desventajas son incremento de la radiación, baja resolución espacial y atenuación por artefactos. Cuando el porcentaje de miocardio no viable es mayor al $40 \%$ por SPECT, la probabilidad de mejorar luego de la revascularización es mucho más baja $^{5}$.

\section{Tomografía por emisión de positrones}

La PET es una herramienta excelente para calcular la viabilidad y supera las limitaciones de la SPECT relacionadas con la atenuación del tejido. El cálculo de la viabilidad miocárdica en áreas de baja perfusión es determinado por la detección del proceso metabólico intracelular intacto, y la 18 fluoro - desoxiglucosa es el agente más usado para el cálculo del metabolismo. Los miocitos usan glucosa en lugar de ácidos grasos libres para la energía durante la isquemia, por lo que áreas con disminución de la perfusión y disminución del metabolismo (balance perfusión-metabolismo) representan cicatrices o muerte del miocardio, mientras que áreas con disminución de perfusión y metabolismo normal (no balance entre perfusión-metabolismo) representan el miocardio viable ${ }^{6}$.

\section{Ecoestrés dobutamina}

El miocardio viable pero disfuncional puede contraerse cuando se expone a inotrópicos, vasodilatadores o ejercicio ligero. La dobutamina es el inotrópico más usado para aumentar la función del ventrículo izquierdo y calcular la viabilidad miocárdica, por lo que la respuesta bifásica, definida como el incremento de la contractilidad a dosis bajas de dobutamina $(<20 \mathrm{mcg} / \mathrm{kg}$ ) y el deterioro con dosis de $40 \mathrm{mcg} / \mathrm{kg}$, indica la presencia de viabilidad miocárdica y 
es el predictor más específico de recuperación luego de la revascularización. La limitación es que es operador dependiente y la adquisición de la ventana puede ser difícil en cierta población. Algunos definen la viabilidad por ecocardiograma estrés con dobutamina, como la presencia de reserva contráctil en más de 5 segmentos disfuncionales ${ }^{7}$.

\section{Resonancia magnética cardiaca}

Es una herramienta reciente para evaluar la viabilidad miocárdica, en donde el aumento o realce de gadolinio es muy útil para cuantificar la fibrosis y visualizar el subendocardio. El gadolinio tiene propiedades paramagnéticas y da señales hiperintensas cuando se acumula, no entra a los miocitos con membrana intacta, pero sí lo hace a miocitos lesionados en el contexto de un infarto agudo y al espacio extracelular cuando hay fibrosis. Luego de alcanzar una estabilidad, este se elimina del miocardio normal, pero permanece en el espacio extracelular en reemplazo de la fibrosis y en los miocitos lesionados. El realce tardío del gadolinio luego de un estado de estrés indica la presencia de una cicatriz miocárdica; en caso de ser mayor al $50 \%$ del grosor de la pared, refleja la ausencia de viabilidad. La RMC también puede diferenciar infartos transmurales de subendocárdicos, medir la reserva contráctil con dosis bajas de dobutamina, así como calcular los volúmenes del ventrículo izquierdo, la cantidad de remodelado, la insuficiencia mitral y los aneurismas, ya que estas son condiciones que pueden afectar significativamente los resultados de la revascularización miocárdica. Las limitaciones principales de esta técnica son sus costos, la disponibilidad limitada, los largos tiempos de realización del estudio y la dificultad de aplicarla en pacientes con dispositivos implantados, aunque recientemente están siendo desarrollados nuevos dispositivos con materiales compatibles (fig. 1) ${ }^{7}$.

\section{Aproximaciones terapéuticas y controversias}

Una vez el cálculo de la viabilidad se realiza, la primera pregunta está relacionada con la cantidad de miocardio viable que se requiere para obtener un beneficio luego de la revascularización. Una mejoría significativa luego de la revascularización se define como un aumento mayor al $5 \%$ en la fracción de eyección del ventrículo izquierdo, lo cual ocurre en el $60 \%$ de los pacientes ${ }^{8}$. La medida de mayor sensibilidad y especificidad para predecir esta mejoría significativa, es que se demuestre que al menos un $25 \%$ del miocardio es viable al usar el ecocardiograma estrés dobutamina ${ }^{9-11}$, y aproximadamente un $38 \%$ usando medicina nuclear y $\mathrm{PET}^{9,10}$. En una estratificación completa del paciente, la presencia y extensión de la cicatriz del miocardio también son predictores importantes, ya que en segmentos discinéticos y acinéticos la ausencia de cicatriz y una extensión transmural menor del $25 \%$ se han relacionado con la recuperación funcional, con un valor predictivo positivo y negativo del $88 \%$ y $89 \%$ respectivamente. ${ }^{12}$.

La controversia en el manejo de pacientes con disfunción del ventrículo izquierdo surge por los datos conflictivos de estudios retrospectivos y estudios aleatorizados controlados. Algunos metaanálisis de estudios retrospectivos muestran que la revascularización comparada con el tratamiento médico óptimo se asocia con menor mortalidad, así que esta medida ha venido aplicándose en pacientes con disfunción del ventrículo izquierdo; sin embargo, ha habido un cambio significativo en la terapia médica en la última década. Los metaanálisis de estudios antiguos acerca del tratamiento médico mostraban que la mortalidad era mayor en pacientes con viabilidad al compararlo con pacientes sin viabilidad $^{3}$, pero metaanálisis más recientes han mostrado que las tasas de mortalidad son equiparables en pacientes tratados médicamente, independientemente de la presencia o no de viabilidad ${ }^{8}$. Lo anterior es un claro reflejo de los avances de la terapia médica en la actualidad, de manera que se ha llegado a considerar que la revascularización miocárdica en la cardiopatía isquémica sólo sería beneficiosa en casos en que la mejoría de la función cardiaca y la sobrevida sean superiores al tratamiento médico. En este punto, cobran importancia otros factores más allá de la viabilidad que pueden mejorar con la revascularización, incluyendo los índices del remodelado ventricular izquierdo como volúmenes y dimensiones, insuficiencia mitral severa, anormalidades de la conducción y la función del ventrículo derecho. Otro factor que puede afectar el pronóstico es el tiempo entre el inicio de la hibernación y la revascularización, ya que el retardo en esta última incrementa el daño irreversible, genera fibrosis y puede llevar a un punto de no retorno del miocardio ${ }^{8}$.

Un estudio reciente en pacientes con y sin viabilidad miocárdica calculada por ecocardiograma estrés dobutamina mostró mejoría de la fracción de eyección del ventrículo izquierdo posterior a la revascularización miocárdica; esta recuperación en pacientes con prueba de estrés negativa para viabilidad, puede interpretarse como la eficacia máxima del tratamiento médico que por sí sola ha mostrado mejorar los desenlaces, o simplemente como la incapacidad del ecocardiograma estrés dobutamina para detectar todo el tejido viable ${ }^{13}$.

Tres estudios aleatorizados, STICH, PPAR-2 y HEART, plantearon la pregunta acerca de la revascularización miocárdica en pacientes con disfunción del ventrículo izquierdo. El STICH comparó terapia médica, con revascularización miocárdica más terapia médica, y revascularización miocárdica con reconstrucción del ventrículo izquierdo más terapia médica en pacientes con disfunción del ventrículo izquierdo. En este estudio se excluyeron pacientes con estenosis del tronco y síntomas de angina, y se encontró que la revascularización no fue mejor a la terapia médica en este tipo de población ${ }^{14}$.

Un subestudio del STICH con seguimiento durante 5 años, mostró resultados relacionados con la viabilidad, y sorpresivamente no encontró ningún beneficio de la revascularización miocárdica sobre la terapia médica, sin embargo, las definiciones pueden influir en el resultado, ya que para la SPECT la definición de viabilidad incluía 11 o más segmentos viables con base a la actividad relativa del trazador, y para el ecocardiograma estrés dobutamina la viabilidad se definía como 5 o más segmentos con función sistólica en reposo alterada pero con manifestaciones de reserva contráctil durante la administración de dobutamina. Otra limitante importante del estudio es que el cruzado fue del $18 \%$ al pasar de manejo médico a revascularización ${ }^{15}$. 

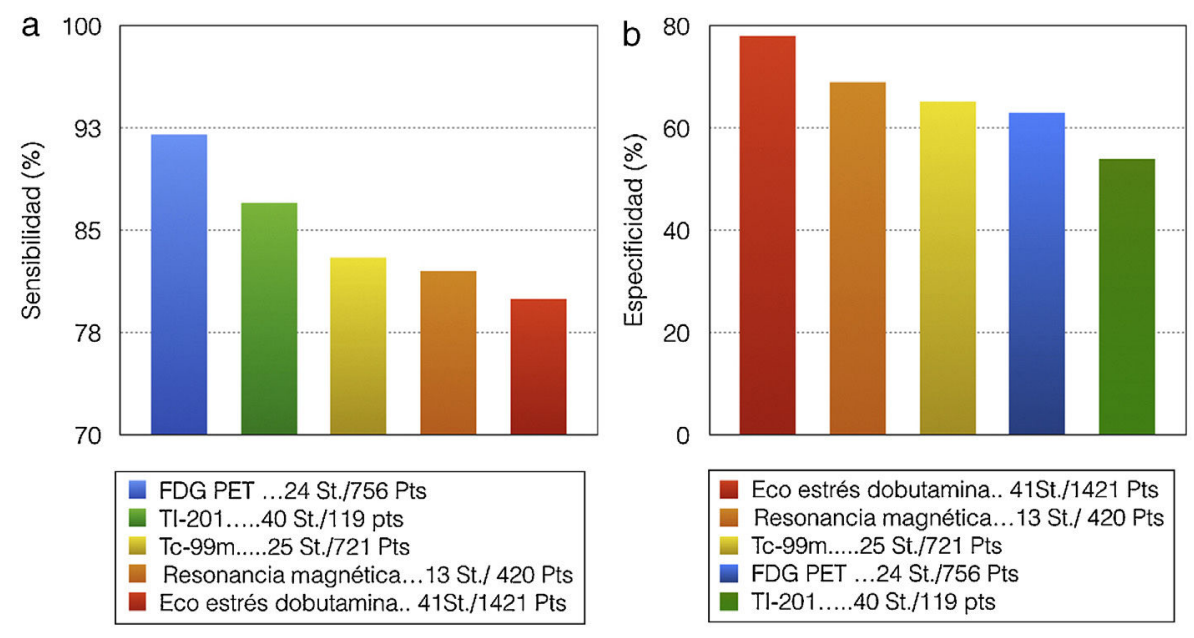

Figura 1 Muestra la sensibilidad y especificidad de las modalidades de imagen actuales usadas para la isquemia miocárdica calculo de la viabilidad. a) Muestra que la PET tiene mejor sensibilidad respecto a las otras pruebas $(p<0,05)$. b) Muestra que el ecocardiograma tiene mejor especificidad al compararlo con otras pruebas $(p<0,05)$. Se infiere que el ecocardiograma estrés dobutamina, al tener la mayor especificidad, tendrá un mayor valor predictivo positivo que los métodos gammagráficos. St.: estudios; Pts: pacientes (modificado de Circulation. 2011;123(4):444-50) ${ }^{7}$.

Los resultados del STICH y el subestudio determinaron que la terapia médica es comparable con la revascularización miocárdica; entonces ¿qué tanto mejora la terapia médica los desenlaces en pacientes con viabilidad? El estudio CHRISTMAS mostró que el carvedilol mejora la fracción de eyección del ventrículo izquierdo en pacientes con viabilidad comparado con placebo, además con mejoría significativa en pacientes que tenían mayor número de segmentos viables. Sumado a lo anterior, los segmentos viables disminuían lentamente en los pacientes que recibían placebo, lo que indica probablemente remodelado y apoptosis de los miocitos mientras en el grupo de carvedilol los segmentos viables permanecieron iguales o aumentaron. En este estudio la media de la FEVI fue del $30 \%$ y la SPECT fue el método utilizado para calcular la viabilidad ${ }^{16}$. Pero lo que más llama la atención es que independientemente del grado de viabilidad, la terapia médica es beneficiosa, mientras la revascularización miocárdica requiere al menos del $20 \%$ de viabilidad para mostrar un beneficio. Otras observaciones interesantes de la terapia médica es que el carvedilol aumenta la $\mathrm{FEVI}$ en pacientes sin reserva contráctil, aún cuando en pacientes con reserva contráctil la mejoría es más robusta y temprana ${ }^{17}$.

Stipac et al., estudiaron el efecto de la revascularización miocárdica a través de la función sistólica del ventrículo izquierdo en pacientes con y sin viabilidad miocárdica para evaluar si los segmentos no viables podrían ser revascularizados. Determinaron que la revascularización en pacientes con áreas de viabilidad detectadas con talio no se asoció con el aumento de la sobrevida a 10 años ni con la mejoría de síntomas de falla cardiaca; sin embargo, sí mostró mejoría en los síntomas de angina ${ }^{18}$.

De otro lado, un estudio controlado aleatorizado evaluó el desenlace de la revascularización miocárdica en pacientes con miocardio viable disfuncional identificado con RMC con estrés dobutamina. En este se definió al miocardio viable como la presencia de más de 4 segmentos con respuesta bifásica en un ventrículo izquierdo disfuncional y con menos del $50 \%$ de realce transmural con gadolinio. Se demostró que en pacientes sin viabilidad la revascularización no fue mejor que la terapia médica, mientras que en pacientes con viabilidad la revascularización miocárdica mejoró significativamente la sobrevida ${ }^{19}$.

\section{Conclusión}

Al evaluar los resultados recientes de los estudios controlados aleatorizados, es posible expresar que el estatus del cálculo de la viabilidad miocárdica en la cardiopatía isquémica permanece sin resolver y por tanto, es preciso definir de manera clara los estadios del remodelado ventricular izquierdo, en los cuales la presencia de viabilidad es benéfica y las etapas en las que el remodelado ventricular es reversible con la revascularización miocárdica. Hoy se considera que la terapia médica y la revascularización miocárdica no son tratamientos competitivos sino complementarios en el tratamiento de la cardiopatía isquémica. En cuanto al método de cuantificación y determinación de viabilidad parece ser que la estrategia de tratamiento basada en la resonancia magnética, puede ser la mejor prueba para evaluar la viabilidad y el remodelado.

\section{Responsabilidades éticas}

Protección de personas y animales. Los autores declaran que para esta investigación no se han realizado experimentos en seres humanos ni en animales.

Confidencialidad de los datos. Los autores declaran que en este artículo no aparecen datos de pacientes.

Derecho a la privacidad y consentimiento informado. Los autores declaran que en este artículo no aparecen datos de pacientes. 


\section{Conflicto de intereses}

\section{Los autores declaran no tener ningún conflicto de intereses.}

\section{Bibliografía}

1. Felker GM, Shaw LK, O’Connor CM. A standardized definition of ischemic cardiomyopathy for use in clinical research. J Am Coll Cardiol. 2002;39:210-8.

2. He J, Ogden LG, Bazzano LA, Vupputuri S, Loria C, Whelton PK. Risk factors for congestive heart failure in US men and women: NHANES I epidemiologic follow-up study. Arch Intern Med. 2001;161:996-1002.

3. Allman KC, Shaw LJ, Hachamovitch R, Udelson JE. Myocardial viability testing and impact of revascularization on prognosis in patients with coronary artery disease and left ventricular dysfunction: a meta-analysis. J Am Coll Cardiol. 2002;39: 1151-8.

4. Desideri A, Cortigiani L, Christen Al, Coscarelli S, Gregori D, Zanco P, et al. The extent of perfusion-F18-fluorodeoxyglucose positron emission tomography mismatch determines mortality in medically treated patients with chronic ischemic left ventricular dysfunction. J Am Coll Cardiol. 2005;46: 1264-9.

5. Pagley PR, Beller GA, Watson DD, Gimple LW, Ragosta M. Improved outcome after coronary bypass surgery in patients with ischemic cardiomyopathy and residual myocardial viability. Circulation. 1997;96:793-800.

6. Tillisch J, Brunken R, Marshall R, Schwaiger M, Mandelkern M, Phelps $M$, et al. Reversibility of cardiac wall-motion abnormalities predicted by positron tomography. $\mathrm{N}$ Engl J Med. 1986;314:884-8.

7. Buckley O, Di Carli M. Predicting benefit from revascularization in patients with ischemic heart failure: imaging of myocardial ischemia and viability. Circulation. 2011;123:444-50.

8. Camici PG, Prasad SK, Rimoldi OE. Stunning, hibernation, and assessment of myocardial viability. Circulation. 2008;117:103-14.

9. Bax JJ, Maddahi J, Poldermans D, Elhendy A, Cornel JH, Boersma $E$, et al. Sequential (201)Tl imaging and dobutamine echocardiography to enhance accuracy of predicting improved left ventricular ejection fraction after revascularization. J Nucl Med. 2002;43:795-802.
10. Bax JJ, Maddahi J, Poldermans D, Elhendy A, Schinkel A, Boersma $\mathrm{E}$, et al. Preoperative comparison of different noninvasive strategies for predicting improvement in left ventricular function after coronary artery bypass grafting. Am J Cardiol. 2003;92:1-4.

11. Hanekom L, Jenkins C, Jeffries L, Case C, Mundy J, Hawley C, et al. Incremental value of strain rate analysis as an adjunct to wall-motion scoring for assessment of myocardial viability by dobutamine echocardiography: a follow-up study after revascularization. Circulation. 2005;112:3892-900.

12. Kim RJ, Wu E, Rafael A, Chen EL, Parker MA, Simonetti O, et al. The use of contrast-enhanced magnetic resonance imaging to identify reversible myocardial dysfunction. N Engl J Med. 2000;343:1445-53.

13. Stipac AV, Stankovic I, Vidakovic R, Putnikovic B, Ilic I, Milicic B, et al. Effect of myocardial revascularisation on left ventricular systolic function in patients with and without viable myocardium: should non-viable segments be revascularised? Heart. 2013;99:1749-54.

14. Velazquez EJ, Lee KL, Deja MA, Jain A, Sopko G, Marchenko $A$, et al. Coronary-artery bypass surgery in patients with left ventricular dysfunction. N Engl J Med. 2011;364:1607-16.

15. Bonow RO, Maurer G, Lee KL, Holly TA, Binkley PF, DesvigneNickens $\mathrm{P}$, et al. Myocardial viability and survival in ischemic left ventricular dysfunction. N Engl J Med. 2011;364:1617-25.

16. Cleland JGF, Pennell DJ, Ray SG, Coats AJ, Macfarlane PW, Murray GD, et al. Myocardial viability as a determinant of the ejection fraction response to carvedilol in patients with heart failure (CHRISTMAS trial): randomised controlled trial. Lancet. 2003;362:14-21.

17. Seghatol FF, Shah DJ, Diluzio S, Bello D, Johnson MR, Cotts WG, et al. Relation between contractile reserve and improvement in left ventricular function with beta-blocker therapy in patients with heart failure secondary to ischemic or idiopathic dilated cardiomyopathy. Am J Cardiol. 2004;93:854-9.

18. Shah PJ, Hare DL, Raman JS, Gordon I, Chan RK, Horowitz JD, et al. Survival after myocardial revascularization for ischemic cardiomyopathy: a prospective ten-year follow-up study. J Thorac Cardiovasc Surg. 2003;126:1320-7.

19. Gerber BL, Rousseau MF, Ahn SA, le Polain de Waroux J-B, Pouleur A-C, Phlips T, et al. Prognostic value of myocardial viability by delayed-enhanced magnetic resonance in patients with coronary artery disease and low ejection fraction: impact of revascularization therapy. J Am Coll Cardiol. 2012;59:825-35. 